\title{
Estudo gastroscópico em eqüinos adultos com suspeita de ulceração gástrica
}

\section{A gastroscopy survey of adult horses with suspicion of gastric ulcers}

\author{
Carla Bargi Belli, ${ }^{*}$ Luís Cláudio Lopes Correia da Silva, ${ }^{* *}$ Wilson Roberto Fernandes ${ }^{\star * *}$
}

\begin{abstract}
Resumo
A ulceração gástrica pode causar uma série de manifestações clínicas em eqüinos adultos, mas ainda faltam conhecimentos sobre essa enfermidade no Brasil. Para avaliar sua importância clínica e alguns fatores associados à etiopatogenia, foram avaliados, através de gastroscopia, 20 eqüinos adultos com suspeita clínica da presença de úlceras gástricas. Concluiu-se que: o número total de animais atendidos com ulceração gástrica foi maior no grupo dos estabulados do que no dos criados a campo. As ulcerações devem ser consideradas como causa de manifestações clínicas (principalmente cólica recorrente), ocorrendo mais na região aglandular ao longo do margo plicatus. Quanto maior o número, a extensão e a profundidade das úlceras, maior a probabilidade de estarem correlacionadas com manifestações clínicas.
\end{abstract}

Palavras-chave: úlcera gástrica, eqüino, gastroscopia.

\begin{abstract}
The gastric ulcer can cause a variety of clinical manifestations in adult horses, but in Brazil, there is still a lack of knowledge of this disease. To assess its clinical relevance and some of the factors associated to etiology and pathogenesis, twenty adult horses with clinical suspicion of gastric ulcers were valued by gastroscopy. It was concluded that the total number of animals attended with gastric ulceration was grated for the stabled ones than for those raised at pasture. Ulcers should be considered as cause of clinical manifestations (mainly recurrent colic), when they occur predominantly in the squamous mucosa along the margo plicatus. The correlation between ulcers and clinical manifestation is higher, if the ulcers appear in a greater number, extension, and depth.
\end{abstract}

Keywords: gastric ulcer, equine, gastroscopy.

\section{Introdução}

As úlceras gástricas são uma desordem prevalente em potros e eqüinos adultos, sendo que, nestes últimos, o impacto econômico pode ser significativo (maior efeito no desempenho e alto custo de tratamento) (Murray, 1994).

Em muitos casos elas são assintomáticas, sem produzir manifestações clínicas evidentes (Murray, 1994; Rose, Hodgson, 1995). Nos adultos, quando as manifestações ocorrem, podem variar de sinais suaves até cólica severa, que pode ser aguda ou recorrente. Com exceção da cólica, há apenas sinais vagos como diminuição do apetite, perda de peso leve ou moderada, depressão, dor após alimentação, bocejos, diarréia, alteração de comportamento e hábitos alimentares, e diminuição do desempenho. Eventualmente podem ocorrer bruxismo e aumento da salivação (Andrews et al., 1999; Murray, 1994; Murray et al., 1999; Rose, Hodgson, 1995).
As úlceras gástricas em eqüinos parecem ser de causa multifatorial. Embora não se saiba qual seu mecanismo exato de formação, várias causas já foram implicadas na etiopatogenia do processo: estresse, intensidade e tipo de trabalho, estabulação, regime alimentar, comportamento, enfermidades concomitantes e uso de antiinflamatórios nãoesteroidais (AINEs) (Murray, 1989; Murray, 1994; Murray, 1997; Murray et al., 1996; Rose, Hodgson, 1995; Sandin et al., 2000).

Em um estudo com 111 eqüinos apresentando desconforto abdominal de duração e severidade variáveis, as úlceras ocorreram em $81 \%$ dos animais. Destes, 31 (28,0 \%) tiveram as úlceras como causa confirmada da cólica, $26(23,4 \%)$ tiveram as úlceras como suspeita da causa da cólica, $17(15,3$ $\%)$ tinham úlceras secundárias a outras desordens e 17 $(15,3 \%)$ tinham úlceras não relacionadas com a cólica. A porção aglandular junto ao margo plicatus foi o local de maior ocorrência de lesões (Murray, 1992a).

\footnotetext{
* Pós-graduação, Departamento de Clínica Médica, Faculdade de Medicina Veterinária e Zootecnia, Universidade de São Paulo, Rua Almeida Maia, 38 ap. 62, São Paulo, SP, CEP 02338-060; cbbelli@usp.br

** Departamento de Cirurgia, Faculdade de Medicina Veterinária e Zootecnia, Universidade de São Paulo, Av. Prof. Dr. Orlando Marques de Paiva, 87, Cidade Universitária, São Paulo, SP, CEP 05508-000; silvalc@usp.br

*** Departamento de Clínica Médica, Faculdade de Medicina Veterinária e Zootecnia, Universidade de São Paulo, Av. Prof. Dr. Orlando Marques de Paiva, 87, Cidade Universitária, São Paulo, SP, CEP 05508-000; wilsonrf@usp.br
} 
No exame gastroscópico de 45 eqüinos de 1 a 24 anos, com história ou sintomas de desconforto abdominal, houve presença de úlceras em $93 \%$. A maioria estava na porção aglandular (fundo), aglandular ao longo do margo plicatus e ao nível da curvatura menor. Em 24 animais os sintomas foram atribuídos às úlceras (Murray, 1989).

Foram avaliados, por gastroscopia, 187 eqüinos, de um a 24 anos de idade, comparando-se os achados de 87 animais com histórico de cólica recorrente ou aguda, hiporexia, perda de condição corporal e/ou diarréia crônica com os de 100 animais sem evidências de problemas gastrintestinais. $O$ número e a severidade das lesões gástricas foram maiores nos animais com manifestações clínicas do que nos assintomáticos (Murray et al., 1989).

Em um estudo clínico, foi realizada gastroscopia em 12 eqüinos com manifestações de desordens gástricas. Úlceras na mucosa aglandular estavam presentes em todos os eqüinos, mas apenas um tinha ulceração na porção glandular. Úlceras solitárias próximas ao margo plicatus foram encontradas em eqüinos com manifestações clínicas leves. Em contraste, ulceração gastroesofágica difusa foi acompanhada por manifestações severas (Dieckmann, Deegen, 1991).

Ainda não se conhece completamente a patogenia da enfermidade e nem a influência real dos fatores predisponentes e desencadeantes (Murray, 1992b). A relação entre o número, tamanho e profundidade das úlceras e as manifestações clínicas ou taxas de cicatrização também não estão determinadas nos eqüinos (Macallister et al.,1997). passaram pelo seguinte esquema de avaliação: anamnese, exame físico, exames laboratoriais (hemograma, bioquímica sérica e coproparasitológico) e exame gastroscópico.

Os eqüinos foram submetidos a jejum antes da gastroscopia pelo tempo mínimo de 18 horas para jejum alimentar e de seis horas para jejum hídrico e foram sedados com aplicação intravenosa (IV) de romifidina (Sedivetâ) ${ }^{1}$, com a dose variando de 0,04 a 0,08 $\mathrm{mg} / \mathrm{kg}$, na dependência da índole do animal.

Para o exame endoscópico foi utilizado um videogastroscópio, marca Karl Storz GMBH \& CO, com 300 centímetros de comprimento útil e 14 milímetros de diâmetro, acoplado a uma fonte de luz de xenon, de 175 W, marca Karl Storz GMBH \& CO.

As úlceras foram classificadas segundo o método proposto por Macallister et al. (1997) (Tabela 1). Assim, para cada porção do estômago (glandular e aglandular) as úlceras receberam duas pontuações, uma para a quantidade e uma para a severidade, resultando num total de quatro pontuações para cada estômago.

Pelo fato desta classificação não avaliar a extensão das úlceras, adotou-se também a identificação aproximada da percentagem da área total de cada porção do estômago afetada pelas ulcerações (<5\%; 5 a 10\%; 10 a 45\%; 50 a $75 \%$; 80 a $100 \%)$. Para a região glandular, que em geral não pode ser visualizada completamente, a área total da mucosa também foi estimada.

Mesmo sendo considerada uma enfermidade prevalente e importante na clínica de eqüinos, há poucas informações a respeito das úlceras gástricas em nosso país, com exceção dos trabalhos de Dearo (1995), Klemm (2000) e Veronezi et al. (2002) os quais a-

Tabela 1 - Classificação das úlceras gástricas, de acordo com o número de lesões e severidade. São Paulo, 2001

\begin{tabular}{cll}
\hline Pontuação & \multicolumn{1}{c}{ Número de Lesões } & \multicolumn{1}{c}{ SeVERIDADE } \\
\hline 0 & sem lesões & sem lesões \\
1 & 1 a 2 lesões localizadas & aparentemente superficial (apenas mucosa) \\
2 & 3 a 5 lesões localizadas & envolvendo estruturas profundas \\
3 & 6 a 10 lesões & múltiplas lesões de severidade variável (1, 2 e/ou 4) \\
4 & $>10$ lesões ou difusa & igual ao 2 mas com aparência ativa \\
5 & - & igual ao 4 com hemorragia ou coágulo de sangue aderido \\
\hline
\end{tabular}

Fonte: MACALLISTER, C. G. et al. (1997, p. 431)

bordaram, respec-

tivamente, a gastroscopia em potros assintomáticos, achados de necrópsia em animais adultos e gastroscopia em eqüinos adultos assintomáticos em treinamento de corrida.

O objetivo deste trabalho foi avaliar a importância clínica das úlceras gástricas em eqüinos adultos, verificando sua ocorrência e severidade em animais com quadro clínico compatível com a enfermidade e a interferência do tipo de criação (a pasto ou estabulado) na mesma.

\section{Material e métodos}

Foram avaliados 20 eqüinos, machos e fêmeas, com idade entre quatro e vinte anos, com peso médio de $424 \mathrm{~kg}$. Todos foram encaminhados ao Hospital Veterinário da Faculdade de Medicina Veterinária e Zootecnia da Universidade de São Paulo por apresentarem suspeita clínica de úlcera gástrica e
De acordo com Macallister et al. (1997), as úlceras ativas são aquelas que têm o fundo hiperêmico ou escurecido com aparência necrótica, sendo que, no início da cicatrização, o mesmo pode tornar-se mais rosado e freqüentemente desenvolver um tecido de aparência de granulação. As úlceras inativas são aquelas que não apresentam estes sinais de inflamação no fundo.

Ao final de toda a avaliação, os eqüinos foram classificados de acordo com a presença ou não de úlceras e a correspondência das mesmas com as manifestações clínicas. Assim, eles puderam ser divididos nos que não apresentavam ulcerações, nos que tinham as úlceras confirmadas como causa única das manifestações, nos que as úlceras foram suspei-

\footnotetext{
${ }^{1}$ Boehringer Ingelheim
} 
tas de causar as manifestações, nos que as úlceras eram secundárias a outros processos e colaboravam para as manifestações ou nos que as úlceras não apresentavam relação com as manifestações. Essa mesma classificação foi usada por Murray (1992a) em sua pesquisa com 91 casos de eqüinos com ulcerações gástricas. Os critérios para definir se as úlceras tinham ou não relação com as manifestações foram a correspondência dos achados com a sintomatologia e a identificação de outras enfermidades associadas ou não.

Os resultados foram analisados por comparação de médias, com p d" 0,05. Apenas para a comparação entre tempo de jejum e percentagem da área gástrica observada foi usado teste de correlação. Utilizou-se para estas avaliações o programa de computador GrafPad InStat ${ }^{\mathrm{TM}}$ (GraphPad Software, versão 3.01, 1998).

\section{Resultados}

Dos 20 animais avaliados (cinco fêmeas e 15 machos), com idade média de 9,9 \pm 4,4 anos, 3 (15\%) eram criados a campo (grupo A) e 17 (85\%) em regime de estabulação (grupo B). Nenhum dos animais apresentou alterações no exame físico geral. Os que foram submetidos a palpação retal, paracentese e/ou sondagem nasogástrica não apresentaram nenhuma alteração nas mesmas.

Entre as manifestações clínicas que motivaram o encaminhamento dos eqüinos para a gastroscopia, as de maior ocorrência foram a cólica intermitente (80\%) e o bruxismo (45\%). Outras manifestações de menor ocorrência foram: emagrecimento (20\%), alteração dos hábitos alimentares $(20 \%)$, sialorréia (15\%), diarréia (15\%), disfagia (5\%) e aerofagia (5\%). A cólica intermitente foi citada como única manifestação em $35 \%$ dos animais, e $55 \%$ deles apresentaram pelo menos duas manifestações.

A maioria dos resultados dos exames laboratoriais estava dentro dos valores normais da espécie ou não apresentava alteração correlacionada com as manifestações clínicas. Apenas dois animais tiveram altos valores de uréia e creatina (fato potencialmente relacionado com a presença e gravidade das úlceras): animal 5 (uréia $=87,00 \mathrm{mg} / \mathrm{dl}$ e creatinina $=$ $5,40 \mathrm{mg} / \mathrm{dl}$ ) e animal 15 (uréia $=117,00 \mathrm{mg} / \mathrm{dl}$ e creatinina $=$ 7,30 mg/dl) do grupo B.

Os demais resultados, em média e desvio-padrão foram: hemácias $\left(7,96 \pm 1,24 \mathrm{milh} / \mathrm{mm}^{3}\right)$, hematócrito $(35,60 \pm 5,81$ $\%$, hemoglobina $(12,46 \pm 2,18 \mathrm{~g} \%), \operatorname{VCM}(44,74 \pm 3,57 \mathrm{fl})$, HCM $(15,63 \pm 1,17 \mathrm{pg}), \mathrm{CHCM}(34,95 \pm 1,06 \%)$, leucócitos $\left(8.225 \pm 2.744 / \mathrm{mm}^{3}\right)$, neutrófilos segmentados $(5.547 \pm 2.676$ $\left./ \mathrm{mm}^{3}\right)$, eosinófilos $\left(74 \pm 101 / \mathrm{mm}^{3}\right)$, basófilos $\left(18 \pm 36 / \mathrm{mm}^{3}\right)$, linfócitos $\left(2.334 \pm 1.035 / \mathrm{mm}^{3}\right)$, monócitos $\left(250 \pm 223 / \mathrm{mm}^{3}\right)$, uréia $(56,35 \pm 25,22 \mathrm{mg} / \mathrm{dl})$, creatinina $(2,12 \pm 1,49 \mathrm{mg} / \mathrm{dl})$, proteína total $(7,24 \pm 1,12 \mathrm{~g} / \mathrm{dl})$, albumina $(3,30 \pm 0,49 \mathrm{~g} / \mathrm{dl})$, AST (184,05 \pm 89,81 U/L), GGT (26,60 \pm 42,27 U/L), bilirrubina total $(1,770 \pm 1,201 \mathrm{mg} / \mathrm{dl})$, bilirrubina direta $(0,238 \pm 0,139$ $\mathrm{mg} / \mathrm{dl}$ ), coproparasitológico (395 $\pm 1.188 \mathrm{OPG})$.

O tempo médio de jejum foi de 22,0 $\pm 2,4$ horas, variando de 18 a 26 horas. A percentagem da mucosa glandular que pôde ser observada variou de 10 a $100 \%$, com média de $51 \pm 22 \%$. Não houve correlação entre estas duas variáveis.

Úlceras esofágicas foram observadas em três animais. 0 animal A-2, cujo histórico revelou recente mudança brusca de manejo (de um pasto em um haras sem realizar nenhum tipo de atividade para um terreno baldio, com alimentação baseada em farelo de milho e feno e colocado para realizar tração de carroça), apresentou ulcerações esofágicas superficiais e ativas (hiperêmicas e hemorrágicas) que se estendiam da porção média do esôfago (aproximadamente a 1,2 metro da narina) até o cárdia. Na área mais cranial do esôfago, as úlceras eram mais lineares (estrias), acompanhando o comprimento do esôfago. Na área mais caudal, as úlceras aumentavam de extensão, tornando-se mais irregulares e chegando a ocupar quase toda a circunferência da luz esofágica. Este achado (úlceras em estrias, que aumentam a área ocupada em direção ao estômago) são típicas de uma esofagite de refluxo.

No eqüino B-15, as úlceras esofágicas eram superficiais, iniciando-se na transição do esôfago cervical para o torácico e se estendendo até a entrada do estômago. Quanto mais próximo ao cárdia, maior a área de lesão da mucosa esofágica. No esôfago do animal B-17 foram observadas apenas regiões pontuais de ulceração superficial, no seu terço médio.

Dentre os 20 animais, 15 (75,0\%) apresentaram ulcerações gástricas, sendo três no grupo criado a campo (100\% do grupo) e 12 no grupo estabulado (70,5\% do grupo). Em seis $(30,0 \%)$ as úlceras foram confirmadas como causa única das manifestações clínicas, em dois $(10,0 \%)$ elas foram suspeitas de ser a causa, em três (15,0\%) eram secundárias a outros processos e colaboravam para as manifestações, em quatro $(20,0 \%)$ as úlceras encontradas não apresentavam relação com as manifestações e cinco $(25,0 \%)$ não apresentavam ulcerações (Tabela 2).

Retirando-se os animais que não apresentaram ulcerações e os que tinham úlceras não relacionadas com as manifestações clínicas, sobram 11 eqüinos. Neles, as percentagens das manifestações clínicas foram: cólica intermitente $(72,72 \%)$, bruxismo $(45,45 \%)$, alterações dos hábitos alimentares $(18,18 \%)$, emagrecimento $(27,27 \%)$, diarréia $(18,18 \%)$, sialorréia $(9,09 \%)$ e disfagia $(9,09 \%)$.

As causas que puderam ser correlacionadas com o aparecimento das ulcerações foram: estresse após competição; alteração abrupta de manejo; estabulação, claudicação e aplicação de AINEs; alteração dentária e má nutrição; uremia; aplicação prolongada de AINEs por alteração locomotora; síndrome cólica; laparotomia e aplicação de AINEs.

A média de idade dos animais sem úlcera gástrica foi de 7,2 $\pm 3,1$ anos e não teve diferença estatisticamente em relação a dos animais com úlcera (10,8 \pm 4,4 anos).

$\mathrm{Na}$ mucosa aglandular, o local onde as úlceras foram observadas com maior freqüência foi o margo plicatus $(75 \%$ do total e $100 \%$ dos animais com ulceração), seguido da curvatura menor (40\% / 53,3\%) (Figura 1) e da curvatura maior (20\% / 26,6\%). Úlceras da mucosa glandular foram observadas em dois animais $(10,0 \%)$.

Foi observada gastrite leve ou moderada na mucosa glandular em 11 animais (55,0\%). Apenas dois animais apresentaram gastrite na mucosa aglandular.

Com relação às observações endoscópicas da mucosa aglandular, a pontuação 0 (sem úlceras) foi encontrada em 5 animais (25,0\%), a 1 em um animal (5,0\%), a 2 e a 3 em 2 animais cada (10,0\%) e a 4 em 10 animais (50,0\%). Apenas o 
Tabela 2 - Relação entre a presença de úlceras e as manifestações clínicas, processos associados às úlceras e causas das manifestações. São Paulo, 2001

\begin{tabular}{|c|c|c|c|}
\hline GRUPO & ANIMAL & $\begin{array}{l}\text { RELAÇÃo ÚLCERA/MANIFESTAÇÕES } \\
\text { CLÍNICAS }\end{array}$ & $\begin{array}{l}\text { PROCESSOS ASSOCIADOS OU } \\
\text { CAUSAS }\end{array}$ \\
\hline \multirow[t]{3}{*}{ A } & 1 & úlcera como causa única confirmada & \\
\hline & 2 & úlcera como causa única confirmada & \\
\hline & 3 & úlcera como causa única confirmada & \\
\hline \multirow[t]{17}{*}{ B } & 1 & úlcera como suspeita & \\
\hline & 2 & sem ulceração & \\
\hline & 3 & úlcera sem relação com manifestações & intoxicação por eucalipto \\
\hline & 4 & úlceras secundárias & alterações dentárias; má nutrição \\
\hline & 5 & úlceras secundárias & uremia \\
\hline & 6 & úlcera como causa única confirmada & \\
\hline & 7 & úlcera sem relação com manifestações & aerofagia \\
\hline & 8 & sem ulceração & \\
\hline & 9 & sem ulceração & \\
\hline & 10 & úlcera sem relação com manifestações & causa não diagnosticada \\
\hline & 11 & úlcera como suspeita & \\
\hline & 12 & sem ulceração & \\
\hline & 13 & úlcera como causa única confirmada & \\
\hline & 14 & úlcera sem relação com manifestações & causa não diagnosticada \\
\hline & 15 & úlcera como causa única confirmada & uremia \\
\hline & 16 & sem ulceração & \\
\hline & 17 & úlceras secundárias & cólica gasosa \\
\hline
\end{tabular}

animal 6 do grupo B teve ulcerações profundas (Figura 2). Em todos os outros as úlceras encontradas foram superficiais. Dos nove animais com área de ulceração acima de 5\%, em oito $(88,8 \%)$ as úlceras apresentaram relação com as manifestações clínicas.

Uma aparência ativa das úlceras foi observada em dez animais (50,0\%). Em relação aos eqüinos onde as úlceras foram comprovadas (como causa única ou associada) ou suspeitas de causar as manifestações clínicas, a aparência ativa das úlceras foi observada em 90,9\% (dez dos 11 animais). A hiperqueratose da mucosa aglandular foi observada em 30,0\% dos animais. Do total de seis animais com hiperqueratose, cinco $(83,3 \%)$ apresentaram ulceração gástrica aglandular.

\section{Discussão}

Em primeiro lugar, é importante verificar que, dos animais encaminhados ao Hospital Veterinário para a avaliação da presença de úlceras gástricas, os criados em regime de estabulação (grupo B) foram em número quase seis vezes maior em relação aos mantidos a campo (grupo $A$ ). E o número de animais com ulceração no grupo $B$ foi quatro vezes maior que no grupo $A$.

Longe de ser uma coincidência, esse resultado, embora possa ser parcialmente um reflexo da maior percentagem de animais estabulados atendidos no HOVET-USP, indica uma influência do sistema de manejo na integridade gástrica dos eqüinos, como verificado em outros trabalhos da literatura sobre a influência do confinamento no desenvolvimento de úlcera gástrica em eqüinos (Murray, Eichorn, 1996; Murray et al., 1999). Logicamente, não se pode dizer que a prevalência das úlceras foi maior nos eqüinos estabulados (para essa afirmação o trabalho não oferece dados suficientes), mas pode-se dizer que a ocorrência das úlceras (relacionada apenas com o número total de animais avaliados) foi maior no grupo estabulado.

É interessante notar também que os três animais criados a campo tiveram as úlceras como causa confirmada das manifestações clínicas. Mesmo sendo um número pequeno de animais, pode-se levantar a possibilidade de que, apesar de eqüinos criados a campo serem menos predispostos a apresentarem úlceras gástricas, quando se suspeita de sua presença é provável que elas realmente estejam presentes e sejam a causa das manifestações clínicas.

Nos animais avaliados no trabalho (adultos), a idade não teve influência sobre o aparecimento de úlceras gástricas. Essa ausência de relação entre idade e presença de úlceras também foi observada em estudo necroscópico em eqüinos adultos (Hammond et al., 1986).

Em relação às manifestações clínicas relacionadas com a presença de úlceras neste trabalho, todas estão de acordo com a literatura consultada.

Muitos pesquisadores (Murray, 1994; Murray et al., 1999; Proudman, Baker, 1994; Rose, Hodgson, 1995) citam o bruxismo como a manifestação mais comum em potros e apenas eventual em eqüinos adultos, estando nestes mais relacionada com o refluxo gastroesofágico, como conseqüência da irritação das mucosas oral e esofágica (Becht, Byars, 1986; Murray et al., 1999). Porém, neste trabalho, ele apareceu como a segunda manifestação mais correlacionada com a presença de úlceras gástricas. Alguns animais com bruxismo não apresentaram ulcerações gástricas como causa das manifestações clínicas, mas sua relação com essa enfermidade em eqüinos adultos não pode ser desprezada.

A manifestação clínica mais correlacionada com úlceras gástricas foi a presença de cólica intermitente, correspondendo à afirmação de Murray (1989) que diz em seu trabalho que a ulceração gástrica deve ser considerada como causa de desconforto abdominal em eqüinos adultos, principalmente se as cólicas forem recorrentes.

A disfagia, única manifestação pouco citada na literatura, ocorreu em apenas um eqüino (animal 2 do grupo A) e estava relacionada com a ulceração esofágica. As úlceras de esôfago e estômago observadas (Figura 3), bastante severas e extensas, foram atribuídas ao estresse provocado por mudanças no manejo do animal. As ulcerações esofágicas, típicas de uma esofagite ulcerativa de refluxo, podem estar relaciona- 


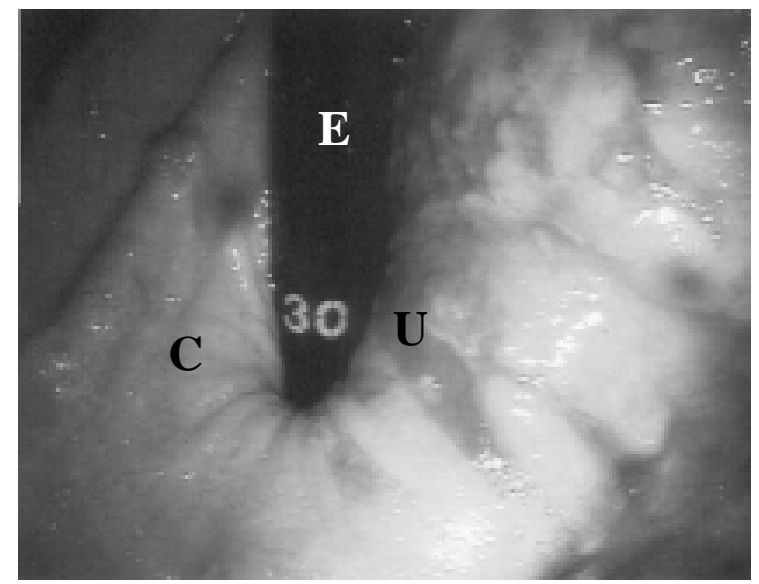

Figura 1 - Úlcera (U) superficial aguda aglandular na região da curvatura menor, junto ao cárdia (C). Endoscópio (E) em retroflexão.

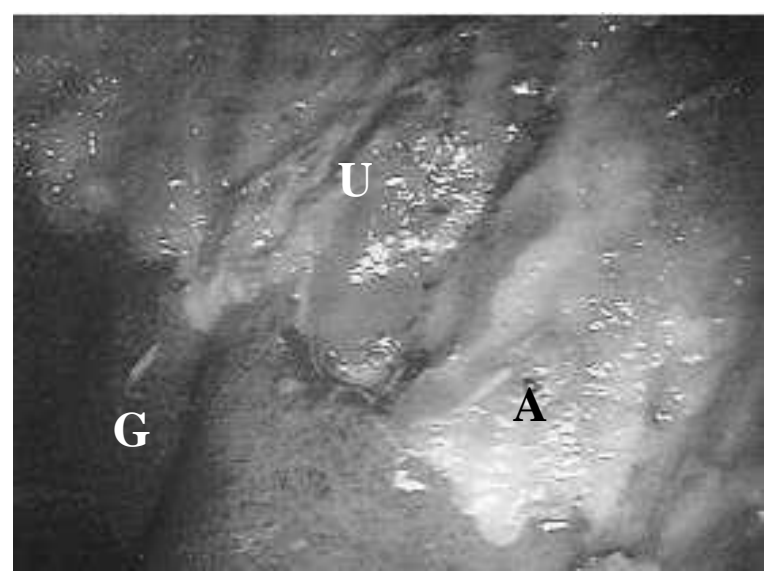

Figura 2 - Úlcera $(U)$ profunda aguda aglandular na região do margo plicatus. A - mucosa aglandular; G - mucosa glandular.

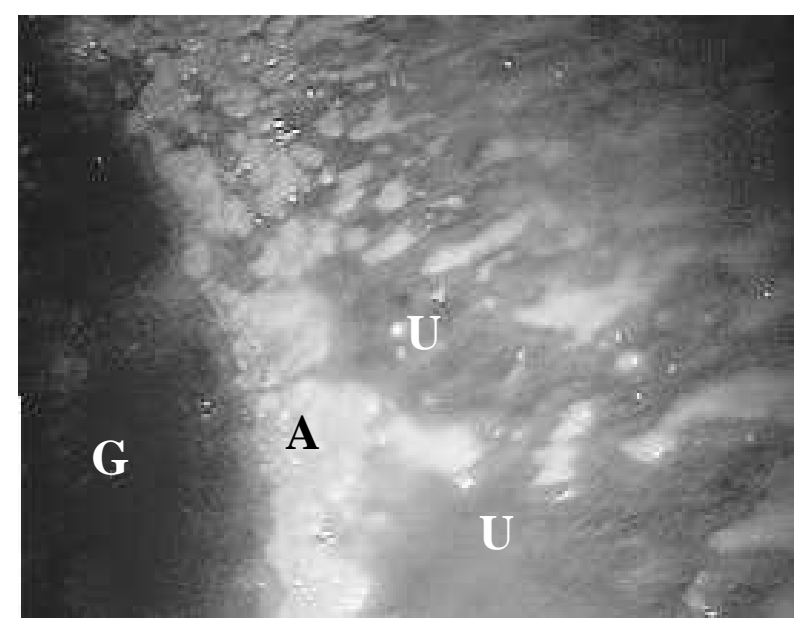

Figura 3 - Úlcera (U) superficial aguda aglandular extensa. A mucosa aglandular; G - mucosa glandular. das não só com a grande extensão das lesões gástricas, como também com o aparente retardo de esvaziamento gástrico neste animal (Becht, Byars, 1986; Murray et al., 1999; Vatistas et al., 1997).

A percentagem média de mucosa glandular observada $(51 \pm 22 \%)$ foi superior aos valores de 30 a $40 \%$ citados na literatura (Murray et al., 1996). A não-visualização completa da mucosa glandular corrobora as afirmações de que eqüinos normais podem apresentar certa quantidade de conteúdo gástrico após jejum prolongado sem que isto indique uma deficiência no esvaziamento gástrico (Brown et al., 1985; Traub-Dargatz, 1992).

Apenas dois animais apresentaram gastrite da mucosa aglandular e, nos dois, as úlceras que a acompanhavam estavam relacionadas com as manifestações clínicas. Mesmo com o pequeno número de animais, é possível sugerir que, ao contrário da gastrite da mucosa glandular, a gastrite aglandular pode ter correlação com as manifestações clínicas apresentadas pelo animal. Já que a hiperqueratose parece ser a principal fonte de proteção da mucosa aglandular (Murray, 1992b), a observação de inflamação desta mucosa não parece ser um achado comum, podendo estar relacionada apenas com os casos mais graves ou então representar novas áreas de aparecimento de úlceras (Murray, 1998).

Neste trabalho, as percentagens de animais onde as úlceras foram confirmadas como causa única das manifestações clínicas, onde foram suspeitas de ser a causa, onde eram secundárias a outros processos, onde não apresentavam relação com as manifestações e onde não ocorriam foram semelhantes aos observados por Murray (1992a) para as mesmas classificações acima.

No que se refere aos animais 10 e 14 do grupo B, a causa das manifestações (cólicas intermitentes nos dois animais) realmente não foi identificada, mas com certeza não estavam relacionadas com a presença das úlceras gástricas. Embora exista uma variação de sensibilidade entre os indivíduos afetados por ulcerações gástricas, as destes dois animais, que eram pequenas e crônicas, com certeza não eram capazes de causar a sintomatologia observada nos mesmos.

Também a presença de uremia nos animais 5 e 15 do grupo B pode ser inicialmente uma confusão na interpretação, visto que em um as úlceras encontradas eram secundárias a essa uremia, e no outro, foram consideradas a causa única das manifestações. Isso é fácil de ser entendido quando se analisa cada caso separadamente. $O$ animal 5 apresentou queixa de bruxismo e diarréia e as úlceras encontradas não eram de gravidade suficiente para ocasioná-las, mas eram secundárias à uremia encontrada neste animal: esta sim, capaz de explicar a sintomatologia encontrada. Já no animal 15, a queixa de cólicas intermitentes não podia ser justificada pela uremia, e sim pelas graves úlceras gástricas e esofágica encontradas no animal. A uremia era a causa das úlceras mas não a causa das manifestações.

Se somarmos os animais deste trabalho onde as úlceras gástricas tiveram participação nas manifestações clínicas (três primeiros grupos), teremos uma ocorrência de $55 \%$ de úlceras como causa de manifestações clínicas. Este valor é bastante semelhante ao encontrado em eqüinos com desconforto abdominal (53,3\%) (Murray, 1989).

Como o desconforto abdominal foi a manifestação clínica mais correlacionada com as úlceras gástricas neste trabalho, pode-se afirmar, em concordância com a literatura consultada, que as mesmas devem ser consideradas como diagnóstico diferencial na clínica de eqüinos, principalmente em casos de cólicas intermitentes.

Uma medida exata das úlceras só pode ser feita por imagens de computador (Murray, Eichorn, 1996). No entanto, este trabalho mostrou que, mesmo uma avaliação subjetiva desta percentagem pode 
ser de grande valia na interpretação dos achados gastroscópicos. A relação observada entre maior extensão das lesões aglandulares e presença de manifestações clínicas indica que esta é uma observação importante a ser feita separadamente durante a gastroscopia em eqüinos, correspondendo aos achados de Dieckmann e Deegen (1991). No entanto, esta observação ainda é pouco utilizada pelos pesquisadores.

A ocorrência de lesões ativas foi alta nos animais onde as úlceras estavam relacionadas às manifestações clínicas, indicando que, em geral, lesões ativas causam mais manifestações que lesões crônicas, concordando com os achados de literatura (Murray et al., 1989).

A presença de hiperqueratose, apesar de poder ser encontrada sem a presença de úlceras (Murray, 1989), neste trabalho mostrou grande associação com as manifestações clínicas. Áreas de descamação gástrica são infreqüentes em animais adultos (Vatistas et al., 1997). Elas foram encontradas em apenas um eqüino deste trabalho, que apresentou as ulcerações gástricas como causa das manifestações clínicas.

Em todos os animais com presença de úlceras gástricas aglandulares, elas estavam localizadas ao longo do margo plicatus, correspondendo ao mencionado na literatura sobre ser esta a localização preferencial de ulcerações gástricas em eqüinos (Hammond et al., 1986; Macallister et al.,1997; Murray, 1994; Murray et al., 1989; Rose, Hodgson, 1995).

Lesões glandulares foram menos comuns, mas em todos os animais onde foram observadas houve correlação com as manifestações clínicas, correspondendo ao que é descrito nos trabalhos de outros pesquisadores (Dieckmann, Deegen, 1991; Furr et al., 1992; Murray, Eichorn, 1996; Vatistas et al., 1997).

O uso de antiinflamatórios foi implicado como causa das úlceras em três animais. Um deles apresentou ulceração da mucosa glandular e nos outros dois foi possível visualizar gastrite moderada e pontos hemorrágicos. Isso confirma as informações da literatura, que afirma que os AINEs, por agi- rem inibindo a biossíntese de prostaglandinas, são a principal causa de ulceração glandular em eqüinos, apesar da interferência de outros fatores (Macallister et al.,1997; Murray, 1992b; Murray et al., 1996; Rose, Hodgson, 1995).

Outros tipos de estresse citados na literatura (Furr et al., 1992; Murray, 1989; Murray, 1994; Murray, 1997; Murray, Eichorn, 1996; Sandin et al., 2000) também puderam ser relacionados com o aparecimento de úlceras neste trabalho: alteração brusca de manejo, enfermidades intercorrentes e competições.

Em concordância com o trabalho de Murray et al. (1989), o número de lesões também foi significativamente maior nos animais com suspeita de úlcera gástrica, principalmente naqueles onde elas estavam relacionadas com as manifestações. Apenas um animal apresentou ulceração profunda, também relacionada com as manifestações clínicas.

É freqüente não haver correlação entre a severidade das úlceras e a severidade das manifestações clínicas em eqüinos adultos (Andrews et al., 1999; Murray, 1994). Isso também pôde ser observado neste trabalho, no entanto, quanto maior o número, profundidade e extensão das úlceras, maior a chance de causarem manifestações clínicas, principalmente se localizadas na mucosa glandular.

\section{Conclusão}

As úlceras gástricas devem ser consideradas como possível causa de manifestações clínicas nos eqüinos adultos, principalmente em casos de cólicas intermitentes. O número total de animais atendidos com ulceração gástrica foi maior no grupo dos estabulados do que no dos criados a campo. Quanto maior o número, extensão e profundidade das ulcerações gástricas em eqüinos adultos, maior a chance de estarem correlacionadas com manifestações clínicas. Por isso, devese utilizar, no exame gastroscópico, uma classificação de úlceras que avalie separadamente a localização, o número, a extensão e a profundidade das lesões, além de outros achados, como a presença de gastrite, pontos hemorrágicos, descamações, hiperqueratose e úlceras esofágicas.

\section{Agradecimento}

À FAPESP (Fundação de Amparo à Pesquisa do Estado de São Paulo).

\section{Referências}

ANDREWS, F.; BERNARD, W.; BYARS, D.; COHEN, N.; DIVERS, T.; MACALLISTER, C.;MCGLADDERY, A.;MERRIT, A.;MURRAY,M.; ORSINI, J.; SNYDER, J.; VATISTAS, N. Recommendations for the diagnosis and treatment of equine gastric ulcer syndrome (EGUS). Equine Veterinary Education, v. 11, n. 5, p. 262-272, 1999.

BECHT, J. L.; BYARS, T. D. Gastroduodenal ulceration in foals. Equine Veterinary Journal, v. 18, n. 4, p. 307-312, 1986.

BROWN, C. M.; SLOCOMBE, R. F.; DERKSEN, F. J. Fiberoptic gastroduodenoscopy in the horse. J. Am. Vet. Med. Assoc., v. 186, n. 9, p. 965-968, 1985.

DEARO, A. C. O. Prevalência de lesões gástricas (úlceras e/ou erosões) em potros assintomáticos da raça quarto de milha. Estudo endoscópico. 1995. 42 f. Dissertação (Mestrado) - Faculdade de Medicina Veterinária e Zootecnia - Universidade Estadual Paulista, Botucatu, 1995.
DIECKMANN, M.; DEEGEN, E. Magenulzera beim Pferd - Klinische und gastroskopische Befunde bei 12 Pferden (1989-1990). Tierärztl Prax, v. 19, n. 4, p. 386-394, 1991.

FURR, M. O.; MURRAY, M. J.; FERGUSON, D. C. The effects of stress on gastric ulceration, $\mathrm{T} 3, \mathrm{TH}$, reverse $\mathrm{T} 3$ and cortisol in neonatal foals. Equine Veterinary Journal, v. 24, n. 1, p. 37-40, 1992.

HAMMOND, C. J.; MASON, D. K.; WATKINS, K. L. Gastric ulceration in mature Thoroughbred horses. Equine Veterinary Journal, v. 18, n. 4, p. 284-287, 1986.

KLEMM, M. Estudo retrospectivo da prevalência de úlceras gástricas em eqüinos necropsiados no "Centro de Anatomia e Anatomia Patológica Romeu Macruz'. 2000. 104 f. Dissertação (Mestrado) - Escola Paulista de Medicina - Universidade Federal de São Paulo, 2000.

MACALLISTER, C. G.; ANDREWS, F. M.; DEEGAN, E.; RUOFF, W.; OLOVSON, S. G. A scoring system for gastric ulcers in the horse. Equine Veterinary Journal, v. 29, n. 6, p. 430-433, 1997. 
MURRAY, M. J. Gastric ulceration in horses with colic. In: ANNUAL CONVENTION OF THE AMERICAN ASSOCIATION OF EQUINE PRACTITIONERS, 34., 1988, San Diego. Proceedings..., 1989. p. 61-68.

. Gastric ulceration in horses: 91 cases (1987-1990). J. Am.

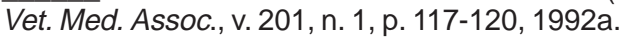

. Gastroduodenal ulceration. In: ROBINSON, N. E. (Ed.) Current $\overline{T h e r a p} y$ in Equine Medicine. 3. ed. Philadelphia: W.B. Saunders Company, 1992b. p. 184-190.

. Gastric ulcers in adult horses. Compend. Contin. Educ. Pract. Vet., v. 16, n. 6, p. 792-794, 1994.

. Gastroduodenal ulceration. In: ROBINSON, N. E. (Ed.). Current $\overline{T h e r a p} y$ in Equine Medicine. 4. ed. Philadelphia: W.B. Saunders Company, 1997. p. 191-197.

MURRAY, M. J. Current concepts in healing of equine gastric ulcers. Equine Practice, v. 20, n. 7, p. 19-20, 1998.

MURRAY, M. J.; EICHORN, E. S. Effects of intermittent feed deprivation, intermittent feed deprivation with ranitidine administration, and stall confinement with ad libitum access to hay on gastric ulceration in horses. American Journal of Veterinary Research, v. 57, n. 11, p. 1599-1603, 1996.

MURRAY, M. J.; GRODINSKY, C.; ANDERSON, C. W.; RADUE, P. F.; SCHMIDT, G. R. Gastric ulcers in horses: a comparison of endoscopic findings in horses with and without clinical signs. Equine Veterinary Journal, p. 68-72, 1989. Supplement 7.

MURRAY, M. J.; SCHUSSER, G. F.; PIPERS, F. S.; GROSS, S. J. Factors associated with gastric lesions in Thoroughbred racehorses. Equine Veterinary Journal, v. 28, n. 5, p. 368-374, 1996.
MURRAY, M. J.; VATISTAS, N. J.; ANDREWS, F. M. Equine gastric ulcer syndrome. Journal of Equine Veterinary Science, v. 19, n. 5, p. 296306, 1999.

PROUDMAN, C. J.; BAKER, S. J. Gastric disease in the adult horse: a clinical perspecive. Equine Veterinary Education, v. 6, n. 4, p. 178184, 1994.

ROSE, R. J.; HODGSON, D. R. Manual Clínico de Equinos. México: Interamericana-McGraw-Hill, 1995.

SANDIN, A.; SKIDELL, J.; HÄGGSTRÖM, J.; NILSSON, G. Postmortem findings of gastric ulcers in Swedish horses older than age one year: a retrospective study of 3715 horses (1924-1996). Equine Veterinary Journal, v. 32, n. 1, p. 36-42, 2000.

TRAUB-DARGATZ, J. L. Laparoscopy, endoscopy and surgical Biopsy. In: ANDERSON, N. V. (Ed.). Veterinary Gastroenterology. 2. ed. Philadelphia: Lea \& Febiger, 1992. p. 67;68.

VATISTAS, N.; SNYDER, J. R.; JOHNSON, B. Adult stomach and duodenum. In: TRAUB-DARGATZ, J. L.; BROWN, C. N. (Ed.). Equine Endoscopy. 2. ed. St. Louis: Mosby, 1997. p. 172-186.

VERONEZI, R. C.; THOMASSIAN, A.; ZANELLA, L. F.; LEGORRETA, G. G. L. Prevalência de úlceras gástricas em eqüinos da raça Puro Sangue Inglês em treinamento para corridas. Revista Brasileira de Ciência Veterinária, v. 9, n. 1, p. 255-257, 2002. Suplemento. Trabalho apresentado no V Congresso Brasileiro de Cirurgia e Anestesiologia Veterinária, Rio de Janeiro, 2002. 